\title{
A tetracycline-inducible CRISPR/Cas9 system, targeting two long non-coding RNAs, suppresses the malignant behavior of bladder cancer cells
}

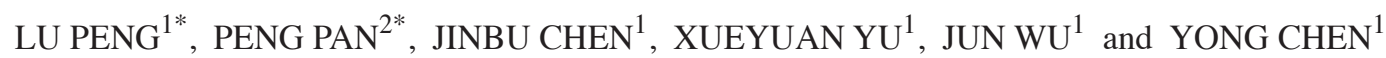 \\ ${ }^{1}$ Department of Clinical Laboratory, Affiliated Brain Hospital of Nanjing Medical University; \\ ${ }^{2}$ Reproductive Medicine Center, Nanjing General Hospital, Nanjing, Jiangsu 210000, P.R. China
}

Received December 16, 2017; Accepted June 28, 2018

DOI: $10.3892 / \mathrm{ol} .2018 .9157$

\begin{abstract}
Clustered regularly interspaced short palindromic repeats (CRISPR) associated protein 9 (Cas9) technology has been applied in varied biological studies, including cancer studies. However, stable mRNA expression of Cas9 has potential risks in future gene therapy. Therefore, in the present study, a tetracycline-inducible switch was used to control the mRNA expression of Cas9. Long non-coding RNAs (lncRNAs) may be important functional regulators in tumor development, including in bladder cancer. RNA was designed to simultaneously target two lncRNAs, PVT1 and ANRIL, which are considered to be bladder cancer oncogenes. The mRNA expression of Cas9 was controlled by doxycycline. Reverse transcription-quantitative polymerase chain reaction revealed that the expression of PVT1 and ANRIL was significantly inhibited by the tetracycline-inducible CRISPR/Cas9 system. Functional assays demonstrated that this system could inhibit proliferation, induce apoptosis and suppress cell migration. Therefore, the tetracycline-inducible CRISPR/Cas9 system was demonstrated to repress the malignant behavior of bladder cancer cells by controlling the expression of Cas9 and simultaneously targeting two oncogenic lncRNAs.
\end{abstract}

\section{Introduction}

Bladder cancer among the most common types of urological neoplasms worldwide (1). The aim of conventional therapies for bladder cancer, including surgery, radiation and chemotherapy, is to eliminate cancer cells. However, adverse effects and treatment failure are common (2-4). Numerous studies

Correspondence to: Professor Yong Chen, Department of Clinical Laboratory, Affiliated Brain Hospital of Nanjing Medical University, 264 Guangzhou Road, Nanjing, Jiangsu 210000, P.R. China E-mail: 13905178354@163.com

*Contributed equally

Key words: tetracycline, CRISPR/Cas9, bladder cancer, IncRNA have focused on the underlying molecular mechanisms of pathogenesis in bladder cancer, and, although long non-coding RNAs (lncRNAs) cannot be translated into proteins, they have emerged as key regulators of the development of bladder cancer (5-7). Therefore, cancer gene therapy via targeting of oncogenic lncRNAs may be a future treatment option.

The lncRNA, PVT1, can promote the progression of various types of tumor, including bladder cancer (8-10). The lncRNA, ANRIL, is also involved in numerous diseases and has been demonstrated to promote DNA methylation, which may be a perinatal marker for subsequent adiposity (11). Overexpression of ANRIL has been reported to accelerate cell invasion and suppress apoptosis in osteosarcoma (12). ANRIL expression is upregulated in bladder cancer and promotes disease progression through the intrinsic pathway (13). Taking into consideration the importance of these two lncRNAs in bladder cancer, they were used as targets in the present study.

Gene editing can alter DNA sequences using nucleases, which act as molecular scissors (14). The clustered regularly interspaced short palindromic repeats (CRISPR)-associated (Cas) protein 9 system combines two components, guide RNA (gRNA) and Cas nuclease (15). This system depends on gRNA for specific cleavage (16). The CRISPR/Cas9 system is considered a promising gene editing tool (17), which can function in various types of cells $(18,19)$. Numerous methods based on this tool have been created and used for cancer study $(20,21)$. It has been revealed that CRISPR/Cas 9 can control gene expression by generating loss-of-function or gain-of-function mutations in oncogenes (22). However, due to potential off-target effects of CRISPR/Cas9, the consistent and safe use of this system remains a challenge. Artificially controlling the 'switch' of this system may reduce the adverse off-target cellular effects.

A tetracycline-inducible element was applied in the present study, consisting of the tetracycline repressor protein (TetR), a specific DNA-binding site, and the tetracycline operator sequence (TetO). TetR is separated from TetO via a conformational change, which is induced by tetracycline or its derivatives, including doxycycline (DOX) (23). The tetracycline-inducible switch controls the expression of Cas9. The nontoxic inducer, DOX, is widely used in preclinical studies (24). Cas9 was efficiently activated when DOX was added to the system. 
Thus, constant expression of Cas9 nuclease could not have been achieved without the presence of DOX.

In the present study, gRNAs were designed to target oncogenes, PVT1 and ANRIL. The objective of the study was to suppress the progression of bladder cancer by targeting multiple sites using the CRISPR/Cas9 system. In addition, the present study aimed to eliminate the off-target effects of this system by utilizing the tetracycline-inducible element. The results indicated that, although all vectors were transfected into cells, the phenotype of the bladder cancer cells was not altered in the absence of DOX. However, when DOX was added, the malignant behavior of bladder cancer cells was significantly inhibited through this tetracycline-inducible CRISPR/Cas9 system. Therefore, this system could efficiently suppress the phenotype of bladder cancer cells and also reduce the side effects of the CRISPR/Cas9 system.

\section{Materials and methods}

Cell lines and cell culture. The human bladder cancer cell lines, T24 and 5637, were obtained from American Type Culture Collection (Manassas, VA, USA). The T24 cells were cultured in DMEM (Invitrogen; Thermo Fisher Scientific, Inc., Waltham, MA, USA) with $10 \%$ fetal bovine serum (FBS; HyClone; GE Healthcare, Chicago, IL, USA). The 5,637 cells were maintained in RPMI-1640 media (Invitrogen; Thermo Fisher Scientific, Inc.) supplemented with 10\% FBS and cultured at $37^{\circ} \mathrm{C}$ in $5 \% \mathrm{CO}_{2}$.

Vectors. A total of 5 gRNA vectors targeting lncRNA PVT1 and 5 targeting lncRNA ANRIL were designed using CRISPR-ERA (http://crispr-era.stanford.edu/). The following gRNA sequences were cloned into a plasmid vector (cat. no. 53188; Addgene, Inc., Cambridge, MA, USA) using the restriction enzymes sites $N d e$ l and $B I P 1$ : PVT1-gRNA1: 5'-TCTCCAGAAGGACAGAATAA-3'; PVT1-gRNA2: 5'-AAA AGA ATT TAATAGACACG-3'; PVT1-gRNA3: 5'-TTGGTGGGGCTTGTGAATC-3'; PVT1-gRNA4: 5'-ACG AGGCCGGCCACGCCACG-3'; PVT1-gRNA 5: 5'-GAT TCACAAGCCCCACCAAG-3'; ANRIL-gRNA1: 5'-GGG GCGCGGCCTCGGCGGAT-3'; ANRIL-gRNA2: 5'-CCG CTCCTCGGCCAAGTCCA-3'; ANRIL-gRNA3: 5'-CGC CGCGGCGCGGGGACTAG-3'; ANRIL-gRNA4: 5'-GCA GCAGCAGCTCCGCCACG-3'; ANRIL-gRNA5: 5'-ACG GCCAACGGTGGATTATC-3'. Tetracycline-inducible Cas9 (vector 1) was purchased from SyngenTech Co., Ltd. (Beijing, China). The vector 2 simultaneously expressing PVT1-gRNA3, PVT1-gRNA4, ANRIL-gRNA2 and ANRIL-gRNA5 was constructed by SyngenTech Co., Ltd, (Beijing, China).

DNA sequencing. An amount of $1 \mu \mathrm{g} / \mathrm{ml}$ DOX was added to the transfected cells and after $48 \mathrm{~h}$ the cells were harvested and genomic DNA was extracted using EasyPure Genomic DNA kit (Beijing Transgen Biotech Co., Ltd., Beijing, China), according to the manufacturer's protocol. DNA sequencing was performed by Sangon Biotech Co., Ltd. (Shanghai, China).

Cell transfection. The propagated vectors were extracted from E. coli using Plasmid Midiprep kit (Promega Corporation, Madison, WI, USA), according to the manufacturer's protocol.
T24 and 5637 cells $\left(2 \times 10^{5}\right)$ were transfected with $2 \mu \mathrm{g}$ vectors for $48 \mathrm{~h}$ per well in 6-well plates using $4 \mu \mathrm{l}$ Lipofectamine ${ }^{\circledR}$ 2000 (Invitrogen; Thermo Fisher Scientific, Inc.) according to the number of cells seeded in the plates. Following transfection, T24 and 5637 cells were used for subsequent experiments immediately.

RNA extraction and reverse transcription-quantitative polymerase chain reaction $(R T-q P C R)$. Total RNA was extracted from $2 \times 10^{6} \mathrm{~T} 24$ or 5637 cells following incubation with TRIzol $^{\circledR}$ (Invitrogen; Thermo Fisher Scientific, Inc.), according to the manufacturer's protocol. Total RNA was reverse transcribed into cDNA using a PrimeScript RT reagent kit with gDNA Eraser (Takara Biotechnology Co., Ltd., Dalian, China), according to the manufacturer's protocol. The mRNA expression levels of PVT1 and ANRIL were measured by RT-qPCR by SYBR ${ }^{\circledR}$ Premix Ex Taq II (cat. no. RR420A; Takara Biotechnology Co., Ltd.) using a Roche LightCycler ${ }^{\circledR}$ 480 Real-Time PCR system. The following thermocycling conditions were used: Initial incubation at $95^{\circ} \mathrm{C}$ for $1 \mathrm{~min}$; 40 cycles at $95^{\circ} \mathrm{C}$ for $30 \mathrm{sec}, 60^{\circ} \mathrm{C}$ for $30 \mathrm{sec}$ and $72^{\circ} \mathrm{C}$ for $30 \mathrm{sec}$, and a final extension step at $72^{\circ} \mathrm{C}$ for $10 \mathrm{~min}$. GAPDH was used as the endogenous control. The following primer pairs were used: PVT1, forward, 5'-GCCCCTTCTATGGGA ATCACTA-3', reverse, 5'-GGGGCAGAGATGAAATCG TAAT-3'; ANRIL, forward, 5'-CAACATCCACCACTGGAT CTTAACA-3', reverse, 5'-AGCTTCGTATCCCCAATGAGA TACA-3'; GAPDH, forward, 5'-CGCTCTCTGCTCCTCCTG TTC-3', reverse, 5'-ATCCGTTGACTCCGACCTTCAC-3'. The comparative $2^{-\Delta \Delta C q}$ method (25) was used to analyze the relative expression of PVT1 and ANRIL. All the experiments were performed at least three times.

Proliferation assay. Cell Counting kit-8 (CCK-8; Beyotime Institute of Biotechnology, Shanghai, China) was used to measure proliferation, according to the manufacturer's protocol. Cells were seeded into a 96-well plate at 5,000 cells per well. Subsequently, 24, 48 or $72 \mathrm{~h}$ after transfection, $10 \mu \mathrm{l}$ of CCK-8 was added to each well and the cells were incubated for $30 \mathrm{~min}$. Absorbance was measured at a wavelength of $450 \mathrm{~nm}$ using an ELISA microplate reader (Bio-Rad Laboratories, Inc., Hercules, CA, USA). All the assays were performed at least in triplicate.

ELISA. A cell death detection ELISA kit (Roche Applied Science, Penzberg, Germany) was used to determined apoptotic rate by quantifying histone-complexed DNA fragments (nucleosomes) in the cytoplasm, according to the manufacturer's protocols. The absorbance was measured at $405 \mathrm{~nm}$ wavelength using a microplate reader (Bio-Rad, Laboratories, Inc.). The experiment was performed $\geq 3$ times.

Cell migration assay. T24 and 5637 cells were seeded in 6 -well plates at $37^{\circ} \mathrm{C}$ and reached $90 \%$ confluence prior to transfection. They were divided into negative control and experimental groups. A mass of $1 \mu \mathrm{g}$ vector 1 and $1 \mu \mathrm{g}$ vector 2 were transfected into the cells and a sterile pipette tip was used to create a wound in the cell layer. After $24 \mathrm{~h}$ of transfection, the migration distance was detected using the software program, HMIAS-2000 (version 2.0; Wuhan 
A

PVT1

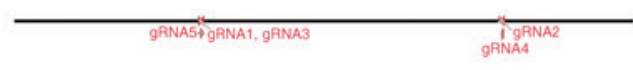

ANRIL

GPNA3 gANA1

\section{C}

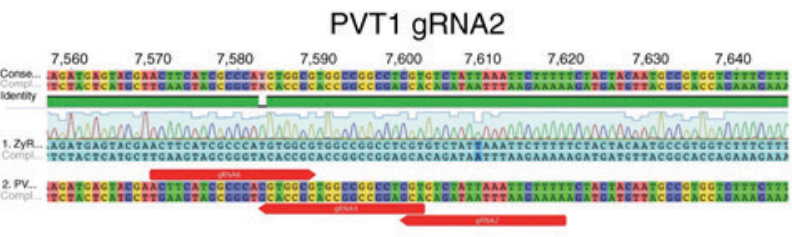

ANRIL gRNA2

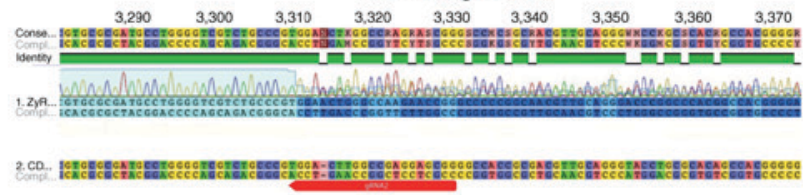

$\mathrm{E}$
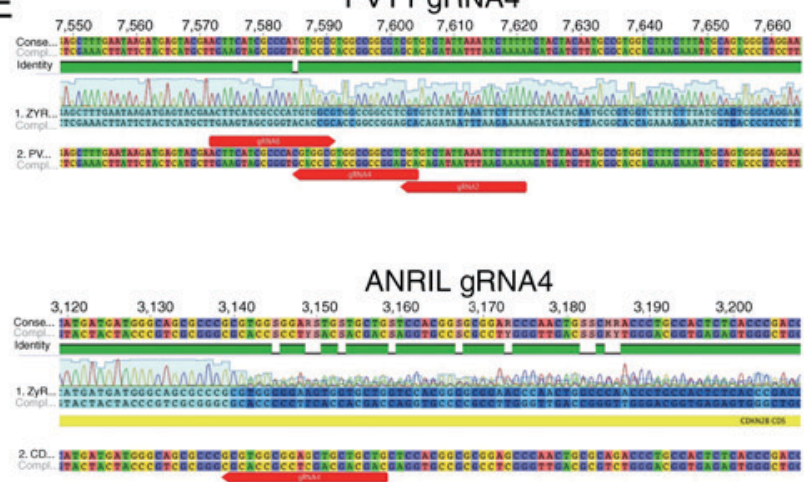

B

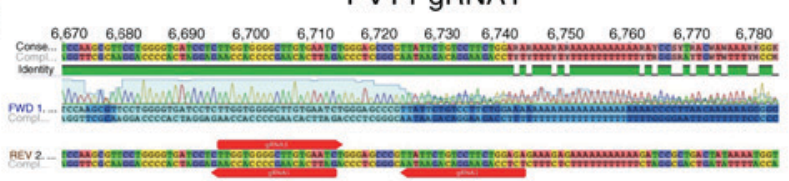

ANRIL gRNA1

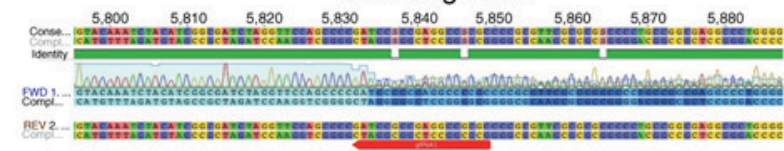

D

PVT1 gRNA3

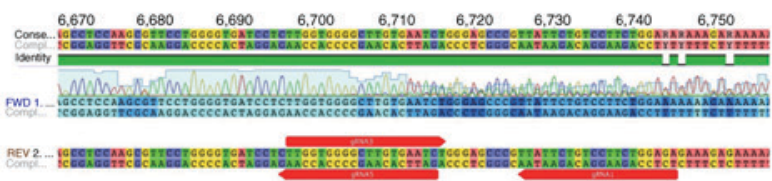

ANRIL gRNA3

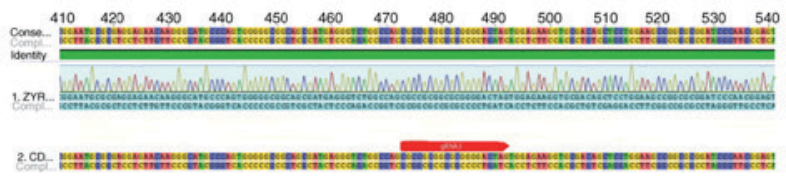

PVT1 gRNA5

$\mathrm{F}$
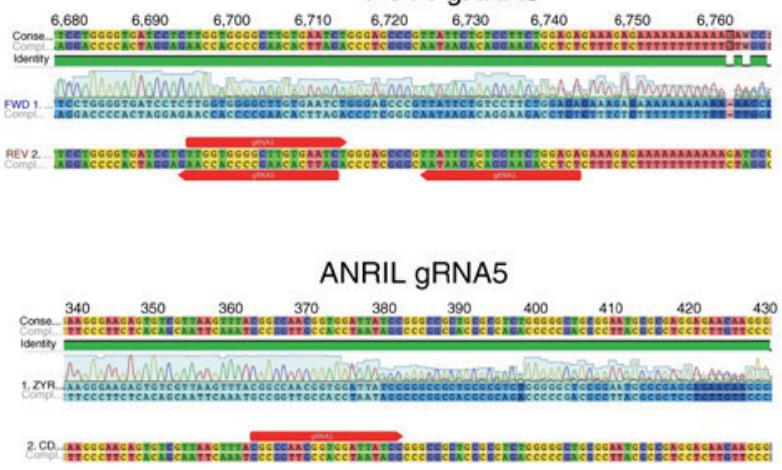

Figure 1. DNA sequencing was performed following transfection of cells with different gRNAs and Cas9. (A) The locations of the gRNA targets on PVT1 and ANRIL. Overlapping peaks were evident when the CRISPR/Cas9 system generated mutations in the PVT1 and ANRIL sequences using (B) gRNA1, (C) gRNA2, (D) gRNA3, (E) gRNA4 and (F) gRNA5. No change was observed in PVT1 targeted by gRNA2. There was no effect on the DNA sequence of ANRIL following transfection with gRNA3/Cas9. gRNA, guide RNA; CRISPR, clustered regularly interspaced short palindromic repeats; Cas9, CRISPR associated protein 9 .

Qianping Imaging Technology Co., Ltd., Wuhan, China). The experiments were repeated $\geq 3$ times.

Western blotting. The transfected cells were washed with PBS and then lysed in radioimmunoprecipitation assay buffer (Beyotime Institute of Biotechnology). The assay was performed as previously described (26). The specific primary antibody against Cas9 (Streptococcus pyogenes) (clone no. D8Y4K) rabbit $\mathrm{mAb}$ (cat. no. 65832) and GAPDH (clone no. D16H11) XP ${ }^{\circledR}$ Rabbit mAb (cat. no. 5174) were purchased from Cell Signaling Technology, Inc. (Danvers, MA, USA; both dilutions, 1:1,000). Incubation with diluted primary antibodies, whilst shaken gently, was at $4^{\circ} \mathrm{C}$ overnight. The peroxidase-conjugated secondary antibody anti-rabbit IgG (cat. no. A0545) was bought from Sigma-Aldrich; Merck KGaA, Darmstadt, Germany $(1: 10,000)$. Incubation with diluted secondary antibody, whilst shaken, at room temperature for $1 \mathrm{~h}$. The experiments were repeated $\geq 3$ times.

Statistical analysis. All statistical analyses were performed using SPSS 20.0 version software (IBM Corp., Armonk, NY, USA). The data are presented as the mean \pm standard deviation. Data was analyzed using Student's t-test or analysis of variance with the Least-Significant-Difference post hoc test. $\mathrm{P}<0.05$ was considered to indicate a statistically significant difference.

\section{Results}

Shearing efficiency of CRISPR/Cas9, analyzed by DNA sequencing. The bladder cancer cells were cultured in 6-well plates and transfected with gRNA and tetracycline-inducible 

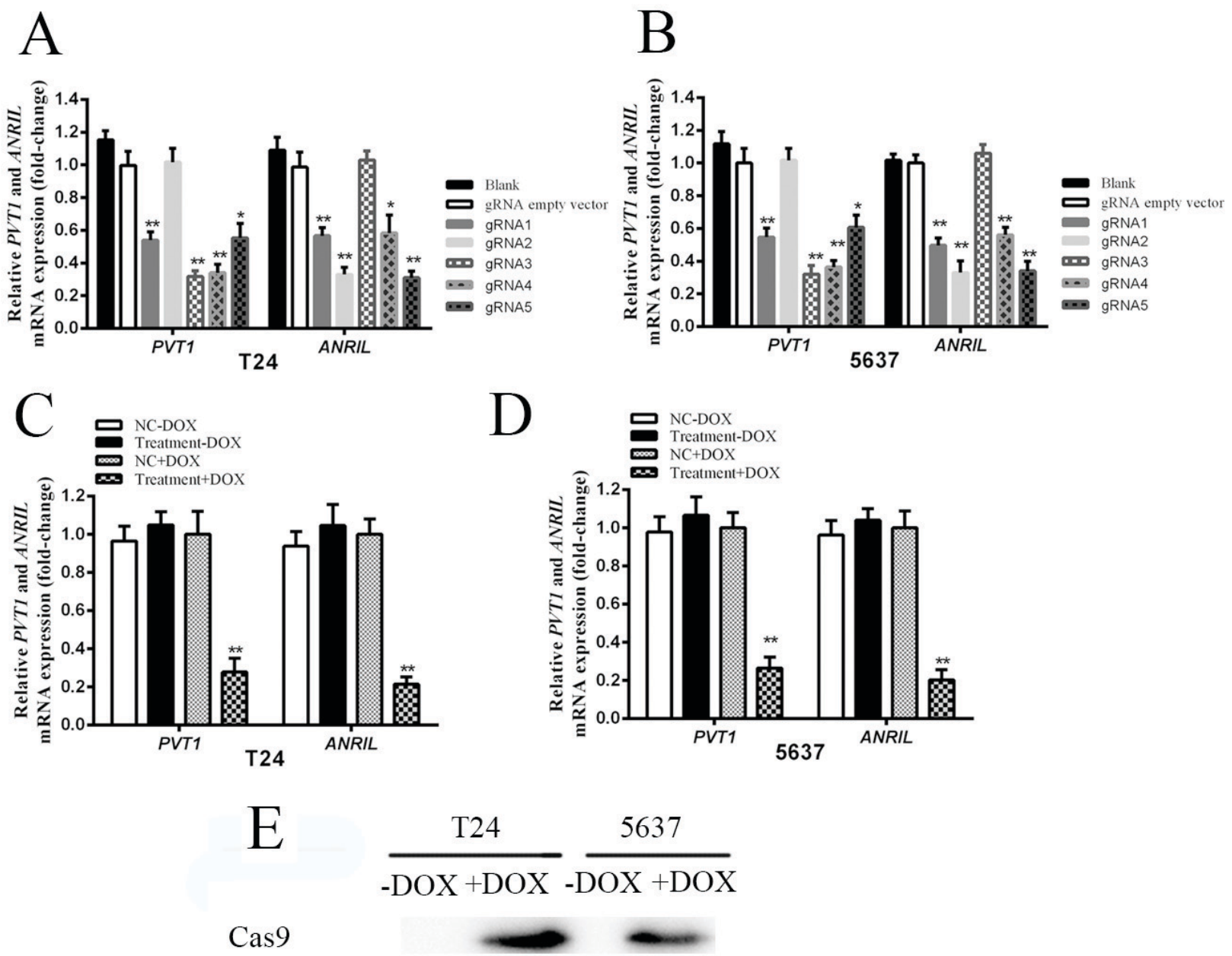

GAPDH

Figure 2. The relative expression levels of PVT1, ANRIL and Cas9 following transfection with tetracycline-inducible CRISPR/Cas9. (A) The expression levels of PVT1 and ANRIL in T24 cells following transfection with different gRNAs and tetracycline-inducible Cas9. (B) The mRNA expression levels of PVT1 and ANRIL in 5637 cells following transfection with different gRNAs and tetracycline-inducible Cas9. The expression levels of gRNA1, gRNA2, gRNA3, gRNA4 and gRNA5 were measured, compared with the gRNA empty vector group. gRNA3 and gRNA4 targeting PVT1, and gRNA2 and gRNA5 targeting ANRIL significantly inhibited PVT1 and ANRIL expression compared with the negative control (gRNA empty vector group) in T24 and 5637 cells. (C) The expression levels of PVT1 and ANRIL were significantly suppressed following addition of DOX in T24 cells, compared with the NC+DOX group cells $(\mathrm{P}<0.01)$. (D) The expression levels of PVT1 and ANRIL were significantly suppressed following addition of DOX in 5637 cells, compared with the NC+DOX group cells $(\mathrm{P}<0.01)$. Error bars represent the mean \pm standard deviation. ${ }^{*} \mathrm{P}<0.05,{ }^{* *} \mathrm{P}<0.01$. (E) In the absence of DOX, Cas9 was not expressed in T24 and 5637 cells. However, following DOX addition, expression of Cas9 was evident. CRISPR, clustered regularly interspaced short palindromic repeats; Cas9, CRISPR associated protein 9; gRNA, guide RNA; DOX, doxycycline.

Cas9 vectors. DOX was used to control mRNA expression of Cas9. Genomic DNA was extracted from the cells $48 \mathrm{~h}$ following transfection. The locations of different gRNAs targeting PVT1 or ANRIL are indicated in Fig. 1A. A total of 5 gRNAs targeting PVT1 and 5 targeting ANRIL were designed. When genomic DNA was excised by CRISPR/Cas9, overlapped peaks were identified in the DNA sequence. Overlapped peaks appeared when mutations were generated in the PVT1 and ANRIL sequences using gRNA1 (Fig. 1B), gRNA2 9 (Fig. 1C), gRNA3 (Fig. 1D), gRNA4 (Fig. 1E) and gRNA5 (Fig. 1F). However, no overlapping peaks were evident when gRNA2 was used to target PVT1 (Fig. 1C). In addition, gRNA3 targeting of ANRIL was not effective in tetracycline-inducible CRISPR/Cas9 system (Fig. 1D). Therefore, the effective gRNAs targeting of PVT1 (gRNA1, 3, 4 and 5) and the effective gRNA-targeting of ANRIL (gRNA1,
2, 4 and 5) may guide the system to excise PVT1 or ANRIL DNA (controlled by DOX).

Suppression efficiency of tetracycline-inducible CRISPR/ Cas9, analyzed by RT-qPCR and western blot analysis. The expression levels of PVT1 and ANRIL in bladder cancer cell lines, T24 and 5637, were measured by RT-qPCR. A total of 5 gRNAs targeting PVT1 and 5 gRNAs targeting ANRIL were designed and subcloned into plasmids. Their effects on PVT1 and ANRIL were detected following transfection of each CRISPR/Cas9 system into cells. Knockdown of PVT1 and ANRIL in T24 and 5637 cells was achieved by 4 gRNAs targeting PVT1 (gRNA1, gRNA3, gRNA4 and gRNA5) and 4 gRNAs targeting ANRIL (gRNA1, gRNA2, gRNA4 and gRNA5) (Fig. 2A and B). The gRNA3 and gRNA4 targeting PVT1, gRNA2 and gRNA5 targeting ANRIL 

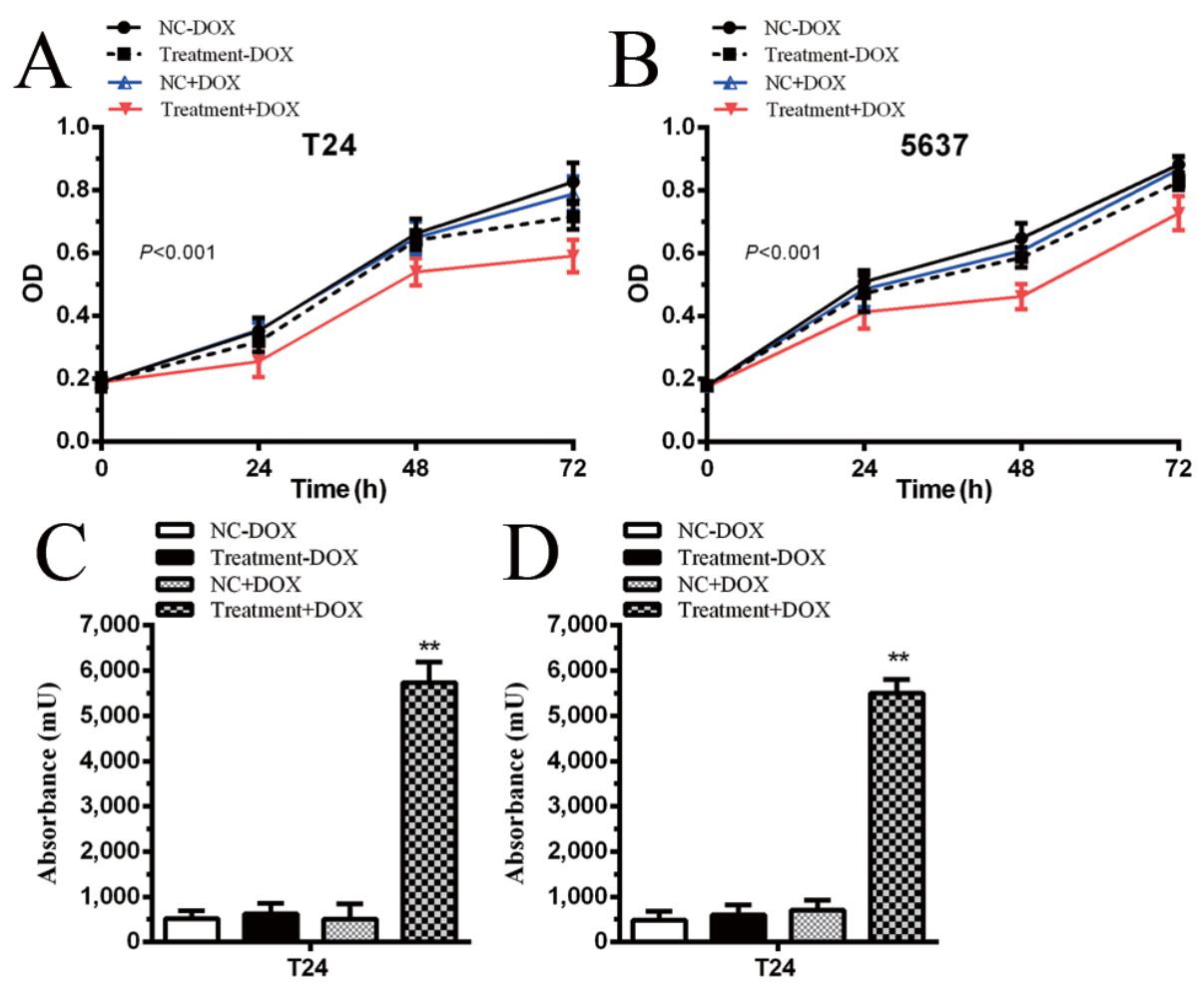

Figure 3. Proliferation was inhibited and apoptotic rate was increased following transfection with tetracycline-inducible CRISPR/Cas9. In the absence of DOX, no significant differences were identified between the NC-DOX and Treatment-DOX groups in (A) T24 cells and (B) 5637 cells (P>0.05). With the addition of $1 \mu \mathrm{g} / \mathrm{ml}$ DOX to the medium, proliferation was significantly inhibited in the Treatment+DOX group compared with the NC+DOX group in T24 and 5637 cells $(\mathrm{P}<0.001)$. In the absence of DOX, no significant differences were identified in apoptotic rate between the NC and Treatment groups in (C) T24 and (D) 5637 cells $(\mathrm{P}>0.05)$. Apoptotic rate was significantly induced in the Treatment+DOX group in T24 and 5637 cells compared with the NC+DOX group $(\mathrm{P}<0.01)$. Error bars represent mean \pm standard deviation. ${ }^{* *} \mathrm{P}<0.01$. CRISPR, clustered regularly interspaced short palindromic repeats; Cas9, CRISPR associated protein 9; DOX, doxycycline; NC, negative control; OD, optical density.

were determined to induce maximal inhibition of PVT1 and ANRIL expression, compared with the negative control in T24 and 5637 cells (Fig. 2A and B). gRNA3 and gRNA4 targeting PVT1, and gRNA2 and gRNA5 targeting ANRIL were selected for further examination. In order to achieve higher inhibition efficiency, the sequences of these four gRNAs were inserted into one vector to achieve simultaneous expression. A quantification analysis was conducted in order to verify the tetracycline-inducible CRISPR/Cas9 system's ability to knock down PVT1 and ANRIL. The mRNA expression levels of PVT1 and ANRIL were significantly suppressed following addition of DOX to T24 cells (Fig. 2C; $\mathrm{P}<0.01$ ) and 5637 cells (Fig. 2D; $\mathrm{P}<0.01$ ). compared with the relative control cells. In conclusion, the expression of Cas9 at the protein level was verified. As indicated in Fig. 2E, Cas9 was expressed in T24 and 5637 cells following DOX addition. However, in the absence of DOX, no expression of Cas9 was evident.

Proliferation is inhibited by tetracycline-inducible CRISPR/Cas 9 in bladder cancer cells. The cells transfected with gRNA vectors and tetracycline-inducible Cas9 vectors were designated as the Treatment group. No significant differences were identified in the negative control (NC) and Treatment groups in T24 and 5637 cells (Fig. 3A and B; P>0.05). When $1 \mu \mathrm{g} / \mathrm{ml}$ DOX was added to the medium, proliferation was significantly inhibited in the Treatment+DOX group in T24 cells compared with the NC+DOX group (Fig. 3A; $\mathrm{P}<0.001$ ) and 5637 cells (Fig. 3B; P<0.001). Proliferation was inhibited by controlling the expression of Cas9 via simultaneously targeting two oncogenic lncRNAs in the bladder cancer cells.

Apoptotic rate is induced by tetracycline-inducible CRISPR/Cas 9 in bladder cancer cells. In the absence of DOX, apoptotic rate was not significantly different between the NC and Treatment groups. However, following the addition of DOX, Cas9 was expressed and CRISPR/Cas9 was able to excise PVT1 and ANRIL. Apoptotic rate was significantly increased in the Treatment+DOX group in T24 cells (Fig. 3C; $\mathrm{P}<0.01$ ) and 5637 cells (Fig. 3D; $\mathrm{P}<0.01$ ) compared with the $\mathrm{NC}+\mathrm{DOX}$ group.

Cell migration was suppressed by tetracycline-inducible CRISPR/Cas 9 in bladder cancer cells. In the absence of DOX, no difference in cell migration was evident between the NC and Treatment groups in T24 (Fig. 4A) and 5637 cells (Fig. 4B). Quantification analysis verified that, in the absence of DOX, no significant differences in cell migration were identified in T24 cells and 5637 cells compared with the control (Fig. 4C and D; $\mathrm{P}>0.05)$. However, with the addition of DOX to the medium, cell migration was repressed in the Treatment+DOX group in T24 and 5637 cells compared with the NC+DOX group. Cell migration was inhibited by almost 50\% in T24 cells (Fig. 4C; $\mathrm{P}<0.01$ ) and $60 \%$ in 5637 cells (Fig. 4D; $\mathrm{P}<0.01$ ). These results suggested that the tetracycline-inducible CRISPR/Cas9 system 
A
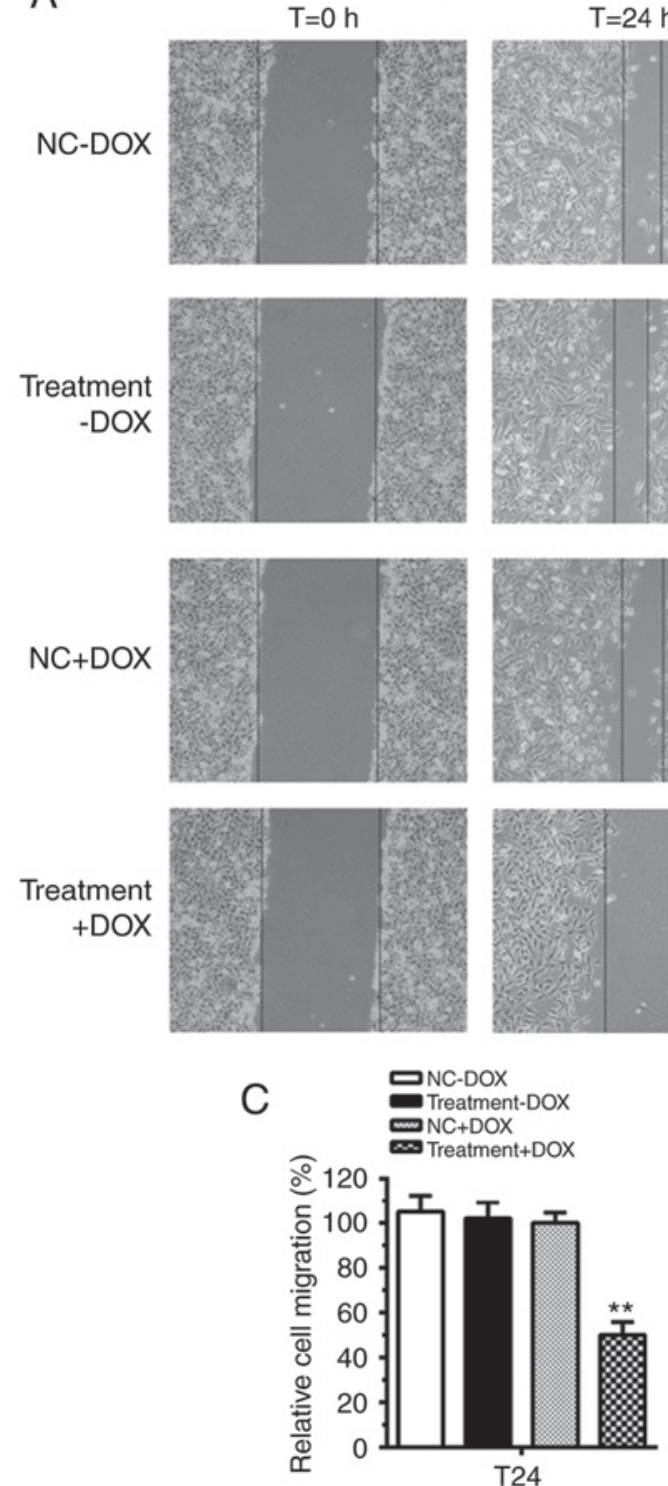
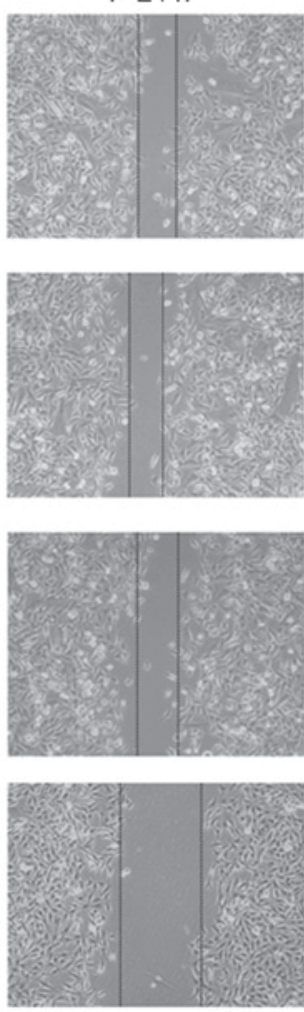

B
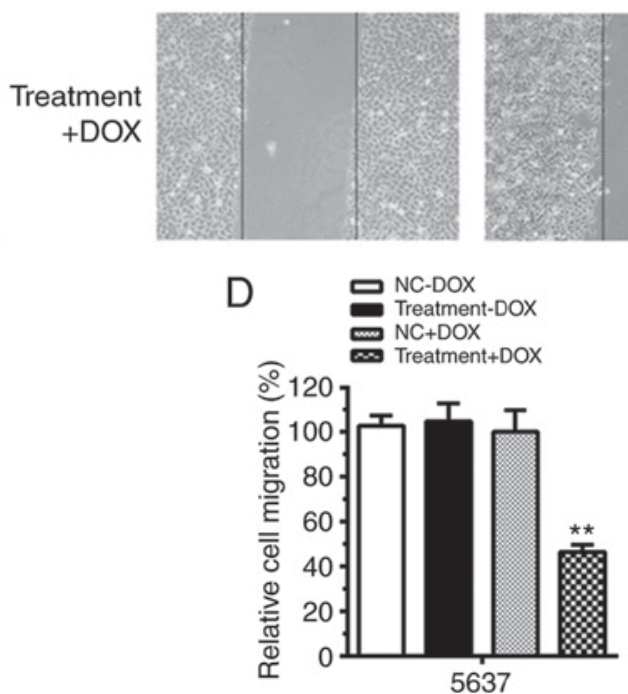

5637
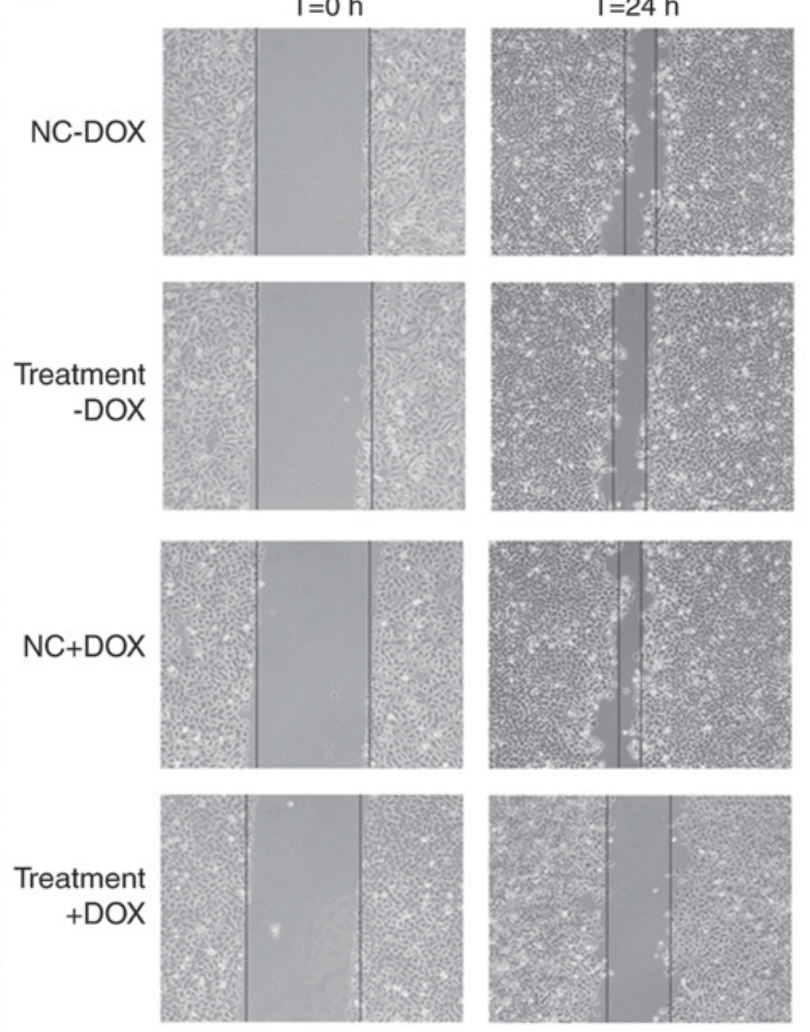

5637

Figure 4. Cell migration was suppressed following transfection with tetracycline-inducible CRISPR/Cas9. (A) In the absence of DOX, no differences in cell migration were identified between NC and Treatment groups in T24 cells. (B) The tetracycline-inducible CRISPR/Cas9 system had no effect on cell migration in the absence of DOX in 5637 cells. (C) In the absence of DOX, quantitative analysis revealed no statistical difference in cell migration between the NC and Treatment groups in T24 cells. With the addition of DOX to the medium, cell migration was significantly inhibited in the Treatment+DOX group compared with the NC+DOX group in T24 cells $(\mathrm{P}>0.05)$. (D) Significant suppression was observed in the Treatment+DOX group compared with the NC+DOX group in 5637 cells. Error bars represent mean \pm standard deviation. ${ }^{* *} \mathrm{P}<0.01$. CRISPR, clustered regularly interspaced short palindromic repeats; Cas9, CRISPR associated protein 9; DOX, doxycycline; NC, negative control; T, time.

could function as an inhibitor of cell migration in bladder cancer cells.

\section{Discussion}

CRISPR/Cas9 is used as a robust genome editing tool to induce specific genomic modifications in mammalian cells $(27,28)$. CRISPR/Cas9 can induce mutations in genomes when multiple gRNAs are integrated with Cas9 in an array (27). This tool has been applied in transcription regulation and gene therapy (15). In a previous study, a catalytically defective Cas9 mutant (dCas9) was created and co-expressed with gRNA to generate a recognition complex, which was indicated to control gene expression at a transcriptional level by interfering with RNA polymerase, elongation and transcription factor binding (29). In addition, CRISPR/Cas9 has been used to target the long-terminal repeat promoter of HIV-1 to inhibit HIV-1 expression in infected human cells (30).

However, there are certain adverse effects of the CRISPR/Cas9 system, including off-target effects, protospacer adjacent motif dependence and gRNA production (15). Researchers have attempted to eliminate the off-target mutations of CRISPR/Cas9 $(31,32)$. The dosage of CRISPR/Cas9 has been demonstrated to affect the off-target effects (15) and, in the present study, a tetracycline switch was used to control the expression of Cas9 to regulate the dosage of CRISPR/Cas9.

Numerous long non-coding RNAs (IncRNAs) serve important roles in the development of different types of cancer 
and may be potential biomarkers (33). Oncogenic lncRNAs in bladder cancer were selected as targets in the present study. With the use of CRISPR/Cas9 to simultaneously knock down $\geq 2$ lncRNAs, higher suppression efficiency may be achieved. Previous studies revealed that overexpression of PVT1 and ANRIL promoted the progression of bladder cancer $(9,13)$. Therefore, these 2 lncRNAs were selected for targeting by gRNAs

In the present study, a tetracycline-inducible CRISPR/Cas9 system was constructed targeting lncRNAs to reduce off-target effects and inhibit the malignant behavior of bladder cancer cells. The results indicated that this tetracycline-inducible system had no effects in the absence of DOX. However, with the addition of DOX, this system could significantly repress the malignant phenotype of bladder cancer cells.

\section{Acknowledgements}

Not applicable.

\section{Funding}

No funding was received.

\section{Availability of data and materials}

The datasets used and/or analyzed during the current study are available from the corresponding author on reasonable request.

\section{Authors' contributions}

LP, PP and JC performed the experiments. JC wrote the paper. XY analyzed the data. JW and YC designed the project. YC provided financial support for the project.

\section{Ethics approval and consent to participate}

Not applicable.

\section{Patient consent for publication}

Not applicable.

\section{Competing interests}

The authors declare that they have no conflicts of interest.

\section{References}

1. Kaufman DS, Shipley WU and Feldman AS: Bladder cancer Lancet 374, 239-249, 2009.

2. Marta GN, Hanna SA, Gadia R, Correa SF, Silva JL and Carvalho A: The role of radiotherapy in urinary bladder cancer: Current status. Int Braz J Urol 38: 144-153, 2012.

3. Racioppi M, Agostino DD, Totaro A, Pinto F, Sacco E, D'Addessi A, Marangi F, Palermo G and Bassi PF: Value of current chemotherapy and surgery in advanced and metastatic bladder cancer. Urol Int 88: 249-258, 2012.

4. Amit D and Hochberg A: Development of targeted therapy for bladder cancer mediated by a double promoter plasmid expressing diphtheria toxin under the control of H19 and IGF2-P4 regulatory sequences. J Transl Med 8: 134, 2010.
5. Droop J, Szarvas T, Schulz WA, Niedworok AC, Niegisch G, Scheckenbach K and Hoffmann MJ: Diagnostic and prognostic value of long noncoding RNAs as biomarkers in urothelial carcinoma. PloS One 12: e0176287, 2017.

6. Berrondo C, Flax J, Kucherov V, Siebert A, Osinski T, Rosenberg A, Fucile C, Richheimer S and Beckham CJ: Expression of the long non-coding RNA HOTAIR correlates with disease progression in bladder cancer and is contained in bladder cancer patient urinary exosomes. PloS One 11: 0147236, 2016.

7. Heubach J, Monsior J, Deenen R, Niegisch G, Szarvas T, Niedworok C, Schulz WA and Hoffmann MJ: The long noncoding RNA HOTAIR has tissue and cell type-dependent effects on HOX gene expression and phenotype of urothelial cancer cells. Mol Cancer 14: 108, 2015.

8. Song J, Wu X, Liu F, Li M, Sun Y, Wang Y, Wang C, Zhu K, Jia X, Wang B and Ma X: Long non-coding RNA PVT1 promotes glycolysis and tumor progression by regulating miR-497/HK2 axis in osteosarcoma. Biochem Biophys Res Commun 490: 217-224, 2017.

9. Zhuang C, Li J, Liu Y, Chen M, Yuan J, Fu X, Zhan Y, Liu L, Lin J, Zhou Q, et al: Tetracycline-inducible shRNA targeting long non-coding RNA PVT1 inhibits cell growth and induces apoptosis in bladder cancer cells. Oncotarget 6: 41194-41203, 2015.

10. Li T, Meng XL and Yang WQ: Long noncoding RNA PVT1 acts as a 'sponge' to inhibit microRNA-152 in gastric cancer cells. Dig Dis Sci 62: 3021-3028, 2017.

11. Lillycrop K, Murray R, Cheong C, Teh AL, Clarke-Harris R, Barton S, Costello P, Garratt E, Cook E, Titcombe P, et al: ANRIL promoter DNA methylation: A perinatal marker for later adiposity. EBioMedicine 19: 60-72, 2017.

12. Wei X, Wang C, Ma C, Sun W, Li H and Cai Z: Retraction note: Long noncoding RNA ANRIL is activated by hypoxia-inducible factor-1 $\alpha$ and promotes osteosarcoma cell invasion and suppresses cell apoptosis upon hypoxia. Cancer Cell Int 17: 60, 2017.

13. Zhu H, Li X, Song Y, Zhang P, Xiao Y and Xing Y: Long non-coding RNA ANRIL is up-regulated in bladder cancer and regulates bladder cancer cell proliferation and apoptosis through the intrinsic pathway. Biochem Biophys Res Commun 467: 223-228, 2015.

14. Clement F, Grockowiak E, Zylbersztejn F, Fossard G, Gobert S and Maguer-Satta V: Stem cell manipulation, gene therapy and the risk of cancer stem cell emergence. Stem Cell Investig 4: 67, 2017.

15. Zhang F, Wen Y and Guo X: CRISPR/Cas9 for genome editing: Progress, implications and challenges. Hum Mol Genet 23: R40-46, 2014.

16. Jinek M, Chylinski K, Fonfara I, Hauer MJ, Doudna A and Charpentier E: A programmable dual-RNA-guided DNA endonuclease in adaptive bacterial immunity. Science 337: 816-821, 2012.

17. Chen X, Janssen JM, Liu J, Maggio I, 't Jong AEJ, Mikkers HMM and Goncalves MAFV: In trans paired nicking triggers seamless genome editing without double-stranded DNA cutting. Nat Commun 8: 657, 2017.

18. Hruscha A, Krawitz P, Rechenberg A, Heinrich V, Hecht J, Haass C and Schmid B: Efficient CRISPR/Cas9 genome editing with low off-target effects in zebrafish. Development 140: 4982-4987, 2013

19. Shen B, Zhang J, Wu H, Wang J, Ma J, Li Z, Zhang X, Zhang $\mathrm{P}$ and Huang $\mathrm{X}$ : Generation of gene-modified mice via Cas9/RNA-mediated gene targeting. Cell Res 23: 720-723, 2013.

20. Chen Y, Zeng S, Hu R, Wang X, Huang W, Liu J, Wang L, Liu G, Cao Y and Zhang Y: Using local chromatin structure to improve CRISPR/Cas9 efficiency in zebrafish. PloS One 12: e0182528, 2017.

21. Huang $\mathrm{X}$, Zhuang $\mathrm{C}$, Zhuang $\mathrm{C}$, Xiong T, Li Y and Gui Y: An enhanced hTERT promoter-driven CRISPR/Cas9 system selectively inhibits the progression of bladder cancer cells. Mol BioSyst 13: 1713-1721, 2017.

22. Sanchez-Rivera FJ and Jacks T: Applications of the CRISPR-Cas9 system in cancer biology. Nat Rev Cancer 15: 387-395, 2015.

23. Vilaboa N and Voellmy R: Regulatable gene expression systems for gene therapy. Curr Gene Ther 6: 421-438, 2006.

24. Goverdhana S, Puntel M, Xiong W, Zirger JM, Barcia C, Curtin JF, Soffer EB, Mondkar S, King GD, Hu J, et al: Regulatable gene expression systems for gene therapy applications: Progress and future challenges. Mol Ther 12: 189-211,2005.

25. Livak KJ and Schmittgen TD: Analysis of relative gene expression data using real-time quantitative PCR and the 2(-Delta Delta C(T)) method. Methods 25: 402-408, 2001. 
26. Zhuang C, Huang X, Zhuang C, Luo X, Zhang X, Cai Z and Gui Y: Synthetic regulatory RNAs selectively suppress the progression of bladder cancer. J Exp Clin Cancer Res 36: 151, 2017.

27. Cong L, Ran FA, Cox D, Lin S, Barretto R, Habib N, Hsu PD, Wu X, Jiang W, Marraffini LA and Zhang F: Multiplex genome engineering using CRISPR/Cas systems. Science 339: 819-823, 2013.

28. Jinek M, East A, Cheng A, Lin S, Ma E and Doudna J: RNA-programmed genome editing in human cells. Elife 2: e00471, 2013.

29. Qi LS, Larson MH, Gilbert LA, Doudna JA, Weissman JS, Arkin AP and Lim WA: Repurposing CRISPR as an RNA-guided platform for sequence-specific control of gene expression. Cell 152: 1173-1183, 2013.

30. Ebina H, Misawa N, Kanemura Y and Koyanagi Y: Harnessing the CRISPR/Cas9 system to disrupt latent HIV-1 provirus. Sci Rep 3: 2510, 2013.
31. Fu Y, Foden JA, Khayter C, Maeder ML, Reyon D, Joung JK and Sander JD: High-frequency off-target mutagenesis induced by CRISPR-Cas nucleases in human cells. Nat Biotechnol 31: 822-826, 2013.

32. Mali P, Aach J, Stranges PB, Esvelt KM, Moosburner M, Kosuri S, Yang L and Church GM: CAS9 transcriptional activators for target specificity screening and paired nickases for cooperative genome engineering. Nat Biotechnol 31: 833-838, 2013.

33. Li J, Li Z, Zheng W, Li X, Wang Z, Cui Y and Jiang X: LncRNA-ATB: An indispensable cancer-related long noncoding RNA. Cell Prolif 50: 2017.

(i) $(9$ This work is licensed under a Creative Commons Attribution-NonCommercial-NoDerivatives 4.0 International (CC BY-NC-ND 4.0) License. 